journal club

\title{
Schulungsprogramm über ein Quartal bei Teens reduziert Diabetesrisiko
}

Fragestellung: Inwieweit kann ein Schulungsprogramm bei Jugendlichen im Alter von 15 Jahren innerhalb von 12 Wochen des Zeitdiabetesrisiko reduzieren?

Hintergrund: In Deutschland geht man von einer hohen Diabetesprävalenz aus. Schaut man aber in die arabischen Länder, ist die Prävalenz um das 3- bis 4-Fache höher. Ein besonderes Problem dabei ist, dass zwei Drittel der Bevölkerung in arabischen Ländern jünger als 25 Jahre sind. Man geht deswegen davon aus, dass in manchen arabischen Staaten und insbesondere Saudi-Arabien im Jahr 2030 eine Diabetesprävalenz von 50 Prozent und mehr erreicht werden könnte. Es gibt sehr viele Treffen, bei denen darüber diskutiert wird, wie man dieser Entwicklung Einhalt gebieten kann. Viele Maßnahmen, die wir in Deutschland oder Europa erfolgreich anwenden, scheitern in diesen Ländern. Ein wichtiger Aspekt ist aber, so früh wie möglich zu agieren und möglichst schon Kinder und Jugendliche zu erreichen. Aiman Bani Salameh hat sich mit seiner Forschergruppe an der Al-Zaytoonah-Universität dieser Frage gewidmet. Ziel war

Originalie

Bani Salameh A, Al-Sheyab N, El-Hneiti $\mathrm{M}$ et al. Effectiveness of a 12-week school-based educational preventive program on weight and fast-ing blood glucose in "at-risk" adolescents of type 2 diabetes mellitus: Randomised controlled trial. Int J Nurs Pract. 2017 Feb 28. doi: 10.1111/ ijn.12528. [Epub ahead of print] hierbei, ob ein Schulungsprogramm bei Jugendlichen (Mädchen und Jungen) helfen kann, das Diabetesrisiko zu reduzieren.

Patienten und Methoden: 401 Jugendliche im Alter zwischen 12 und 18 Jahren (durchschnittlich 15,3 Jahre) wurden in zwei Schulen in Ir- bit in Jordanien in einer kontrollierten randomisierten prospektiven Studie untersucht. Die Teilnehmer wurden zufällig in eine Interventions- und Kontrollgruppe aufgeteilt, alle Teilnehmer mussten an den Untersuchungstagen am Morgen nach 8 Stunden Nüchternphase erscheinen. Die medizinischen Parameter wurden erhoben (Körpergewicht, -fett, Glukose und soziodemografische Kriterien). Die Teilnehmer in der Interventionsgruppe erhielten über 12 Wochen ein Schulungsprogramm, das unterschiedliche Lernmethoden anwandte (Vorträge, Diskussionen, Workshops, Gruppenarbeiten), wobei den Teilnehmern das Thema gesunde Ernährung und Bewegung näher gebracht werden sollte. Die Teilnehmer erhielten elektronische Schrittzähler und sollten Ernährungstagebücher führen. Nach 12 Wochen wurde der Status wie izu Beginn erhoben. Die Kontrollgruppe lauschte nur einem Vortrag über gesunde Ernährung. In beiden Gruppen wurden die Eltern an den Interventionen beteiligt.

Ergebnisse: Die Intervention hat einen Effekt gehabt. Die Jugendlichen in der Interventionsgruppe reduzierten ihr Körpergewicht um 2,3 kg und verbesserten den Nüchternblutzuckerspiegel um $5,02 \mathrm{mg} / \mathrm{dl}$. Die Teilnehmer in der Kontrollgruppe nahmen 800 g zu und ihr Nüchternblutzucker verschlechterte sich um 2,3 mg/ dl. Die Ergebnisse waren gleichermaßen bei Jungen und Mädchen festzustellen, obwohl die Jugendlichen getrennt nach Geschlecht interveniert wurden. Es zeigte sich, dass die männlichen Jugendlichen einen stärkeren Effekt bei körperlicher Aktivität hatten, die weiblichen Jugendlichen allerdings mit einer Ernährungsumstellung und besserer körperlicher Aktivität gleichermaßen gut umgehen konnten. Weiterhin zeigte sich am Ende der Studie, dass das Interesse bei weiblichen Jugendlichen höher war, die Umstellung fortzuführen, als bei den männlichen Jugendlichen.

\section{- Kommentar Prof. Dr. med. Peter E. H. Schwarz}

\section{Gruppendynamik ist ein wichtiger Faktor}

Die Ergebnisse sind toll. Alle Kinder hatten ein erhöhtes Risiko für das metabolische Syndrom und Diabetes mellitus. Mit einer relativ einfachen und niedrigschwelligen Intervention konnte aber ein guter Effekt erzielt werden. In der Intervention waren die Jugendlichen nicht zwingend sportlich aktiv, sondern sollten einfach über eine höhere Schrittzahl körperlich aktiv sein und in der Familie eine gesunde Ernährungsumstellung versuchen. Das hat in der Interventionsgruppe funktioniert. Ein schlechtes Zeichen ist allerdings, dass sich die Situation in der Kontrollgruppe selbst in der kurzen Zeit und trotz allgemeiner Information verschlechtert hat. Das ist häufig in den arabischen Ländern zu beobachten, dass Jugendliche in dieser Altersgruppe sehr schnell an Gewicht zunehmen - häufig assoziiert mit einem konstant steigenden Fast-Food-
Konsum. Die Intervention war bestechend einfach: Schulungen, Diskussionsrunden, Gruppenarbeiten, also vorwiegend interaktiv. Vermutlich war der Effekt, dass die Gruppe an sich teilgenommen hat, ein starker Motivator in der Interventionsgruppe - dieser hat in der Kontrollgruppe komplett gefehlt. Wir lernen also, dass auch kurze Kerninterventionen, die auf Gruppen und interaktive Lernmethoden setzen, effektiv sein können und Risikokindern helfen, Gewicht und Nüchternblutzucker zu reduzieren.

Prof. Dr. med. Peter E. H. Schwarz

Med. Klinik III, TU Dresden und Paul Langerhans Institut Dresden 